\title{
A Dynamic Channel Allocation Technique Based on Hopfield Neural Networks
}

\author{
Enrico Del Re, Senior Member, IEEE, Romano Fantacci, Senior Member, IEEE, and Luca Ronga, Member, IEEE
}

\begin{abstract}
The interest in global spectrum allocation techniques is growing with the always increasing spectrum demand for mobile communications. However, the best algorithms suffer from high computational times that reduce the possibility of a practical implementation. This paper deals with a dyoamic channel allocation (DCA) technique based on an energy function whose minimization gives the optimal allocation. Due to the particular formulation of such an energy function, the minimization can be performed by a Hopfield neural network for which a fast hardware implementation has been recently proposed in the literature. The performance of the proposed DCA technique is here derived by computer simulations. Comparisons with a classical fixed allocation technique (FCA) and a different DCA technique are shown to highlight the better performance of the proposed DCA technique.
\end{abstract}

\section{INTRODUCTION}

$\mathbf{I}$ $\mathrm{N}$ a cellular radio system, the territory is divided into hexagonal cells, with each cell being served by a base station located at its center. Each mobile user needs a channel to communicate with the base station of the cell the user belongs to. ${ }^{1}$ In this paper, we refer to a channel as a generic communication resource, which can be alternatively a radio channel in a specified band for a frequency-division multiple access, a code for a code-division multiple access, or a time slot for a time-division multiple access.

The same channel can be simultaneously used in different cells at a suitable distance $(D)$. The minimum distance between cells in which the use of the same channel is possible (reuse distance) depends on the level of the co-channel interference accepted in order to allow reliable communications.

The channel assignment to the cells can be performed resorting to the FCA technique or to the DCA technique [2]. In the case of the FCA technique, a set of channels is permanently assigned to each cell. The same set of channels is reused in cells at a distance $D$ away. The basic FCA concept implies that a new call generated in a cell can only be served by an available channel belonging to the set of channels assigned to that cell. If no channel is available, the call is blocked and lost. The number of channels permanently allocated to each cell is equal to $M / K$, where $M$ is the number of the system resources and $K$ the reuse factor [2].

Manuscript received September 8, 1994; revised May 8, 1995. This work was performed with the financial support of ASI and MURST.

The authors are with the Dipartimento di Ingegneria Elettronica-Università di Firenze, Firenze, Italy.

Publisher Item Identifier S 0018-9545(96)00189-2.

${ }^{1}$ Actually, for bidirectional communication, each mobile user needs two channels: the first for the mobile-to-base station link and the second for the base station-to-mobile link. These two channels are assigned always in pairs so in our study we can consider only one link.

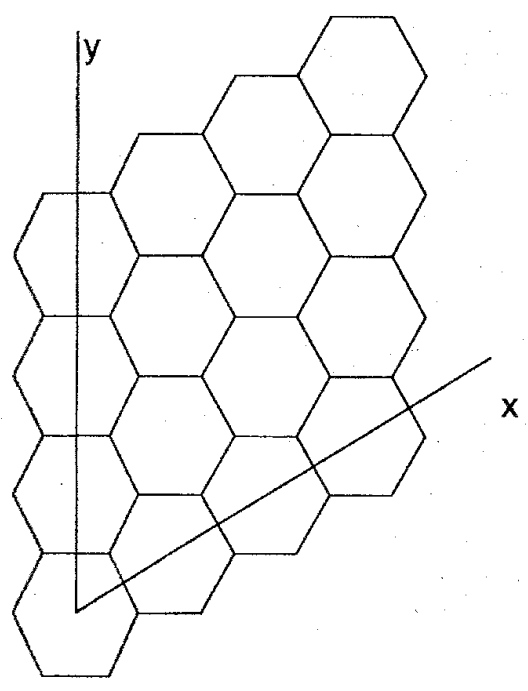

Fig. 1. Cellular network model.

Differently from the FCA technique, in the DCA technique the channels are not permanently assigned to the cells. In this case, channels are assigned on a call-by-call basis in order to obtain a better performance and a narrower spectrum utilization, especially under nonuniform traffic load conditions.

\section{The PRoposed DCA TECHNIQUE}

In order to make each channel assignment technique suitable for application in actual cellular systems, we should limit the number of operations to be performed when a new call arrives. In this paper we assume that the rearranging operations must be carried out only in the cell involved in the new arrival. Raymond [3] proposed a channel allocation algorithm that rearranges the assignment channels in the whole network every time a new call arrives. This technique permits to lower the blocking probability at the expense of an excessive increase of the implementation complexity.

The topological model considered in this paper is a group of hexagonal cells, as shown in Fig. 1.

The number of cells along the $x$-axis is equal to the number of those along the $y$-axis. The "number of cells in a side" is considered as a parameter as well as the "number of available channels" for the whole cellular structure.

We assume that the interference order is $I$, i.e., a cell $\mathrm{C}$ interferes with all the cells $(\mathrm{X})$ belonging to $I$ rings centered in $\mathrm{C}$, as shown in Fig. 2. 


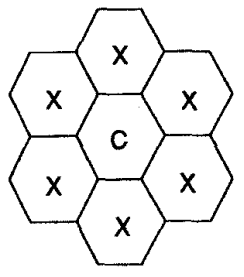

$I=1$

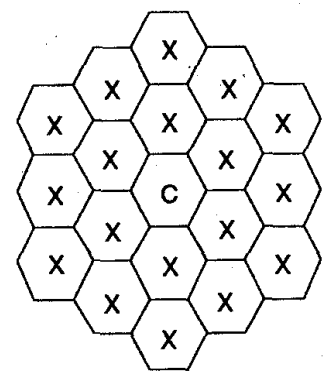

$\mathrm{I}=2$
Fig. 2. Interfering cells.

It is not possible to use simultaneously the same channel in two interfering cells. This condition cannot be violated and so we refer to it as a hard condition. Whenever the channel selected according to a suitable assignment algorithm does not satisfy this constraint, the corresponding service request (new call) is blocked. Other conditions, named soft conditions, have also been provided. Soft conditions differ from hard ones as they can be violated at the expense of a slight decrease of the performance of the allocation algorithm.

The most important soft conditions proposed are the packing condition and the resonance condition.

With the packing condition, assignment solutions that tend to use the minimum number of channels to satisfy the global channel demand are preferred. The impact of this condition on the assignment is to prefer channels already used in other cells, without violating any hard condition. If more choices are possible, channels used in the nearest cells are taken into account. We are going to explain later how to translate this condition and the subsequent ones into terms of a quadratic energy function to minimize.

With the resonance condition, we tend to assign the same channels to the cells that belong to the same reuse scheme, obtained by jumping from one cell to another with steps of length exactly equal to the reuse distance, as shown in Fig. 3.

These conditions tend to give an optimum assignment in the presence of a uniform distribution of incoming calls among the cells. When a nonuniform traffic is present, this condition still seems to perform well.

Others soft conditions are formulated in order to effect the following:

- Assign, where possible, the same channels assigned before, i.e., limiting the infracell rearranging;

- Try to assign the exact number of channels requested in the cell involved in a new arrival (or the termination of a call). A violation of this condition means the impossibility of serving an incoming call that is obviously blocked.

All these conditions lead to the definition of a quadratic energy function, discussed below. In performing our analysis the following definitions are assumed:

$\mathrm{CE}$ total number of cells in the system $\mathrm{CH}$ number of channels available to the system
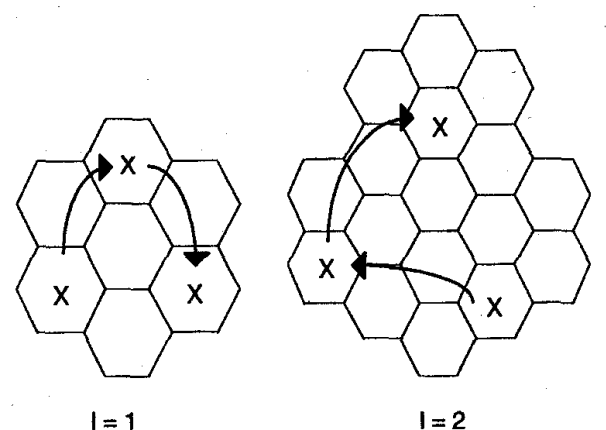

Fig. 3. Reuse schemes.

$\begin{array}{ll}A_{i, j} & \begin{array}{l}\text { an element of the allocation table } \\ \text { whose value is } 1 \text { if channel } j \text { is }\end{array} \\ & \text { allocated to cell } i(i=1, C E \text { and } \\ & j=1, C H), 0 \text { otherwise } \\ & \text { cell involved in a new arrival or a } \\ & \text { termination of a call } \\ i^{*} & \text { number of requested channels at cell } i^{*} \\ \text { Traf }\left(i^{*}\right) & \begin{array}{l}\text { assignment of the cell of interest, i.e., } \\ \text { the variables of the assignment } \\ V_{i^{*}}, j\end{array} \\ \text { problem } \\ \text { function generating a value of } 1 \text { if } \\ \text { cells } i \text { and } i^{*} \text { are interfering according } \\ \text { to the previous definitions, } 0 \text { otherwise } \\ \text { determines the distance between cells } i \\ \text { and } i^{*} \text { normalized to the intercenter } \\ \text { distance between two adjacent cells } \\ \text { value of } 1 \text { if cells } i \text { and } i^{*} \text { belong to } \\ \text { the same reuse scheme defined before, } \\ \text { O otherwise. }\end{array}$

We can define the energy function associated with the cellular network under consideration as follows:

$$
\begin{aligned}
E= & \frac{A}{2} \cdot \sum_{j=1}^{C H} \sum_{\substack{i=1 \\
i \neq i^{*}}}^{C E} V_{i^{*}, j} \cdot A_{i, j} \cdot \operatorname{Interf}\left(i, i^{*}\right) \\
& +\frac{B}{2} \cdot\left[\sum_{j=1}^{C H} V_{i^{*}, j}-\operatorname{Traf}\left(i^{*}\right)\right]^{2} \\
& -\frac{C}{2} \sum_{j=1}^{C H} \sum_{\substack{i=1 \\
i \neq i^{*}}}^{C E} V_{i^{*}, j} \cdot A_{i, j} \cdot \frac{\left[1-\operatorname{Interf}\left(i, i^{*}\right)\right]}{\operatorname{Dist}\left(i, i^{*}\right)} \\
& -\frac{D}{2} \cdot \sum_{j=1}^{C H} V_{i^{*}, j} \cdot A_{i^{*}, j} \\
& +\frac{F}{2} \cdot \sum_{j=1}^{C H} \sum_{\substack{i=1 \\
i \neq i^{*}}}^{C E} V_{i^{*}, j} \cdot A_{i, j} \cdot\left[1-\operatorname{Res}\left(i, i^{*}\right)\right] .
\end{aligned}
$$

The first term adds a positive constant to the energy function if there are some interfering cells using the same channels; the second term is positive if the requested number of channels has not been assigned to cell $i^{*}$; with the third term, we fulfill the packing condition as stated before; the fourth term lowers the value of the energy function if the actual assignment is 
equal to the previous one; and the fifth term accomplishes the resonance condition.

Constants $A, B, C, D$, and $F$ are determined in order to decide which are the conditions that can be violated and in which order, as will be shown later.

Every time a new call arrives (or ends) in a cell $i^{*}$, the algorithm searches for a pattern of 1 's and 0 's that, when substituted to $V_{i^{*}, j}$, minimizes the energy function $E$. The best pattern found, i.e., corresponding to the lowest value of the energy function, represents the solution to the DCA problem only if it satisfies the channel demand condition. On the contrary, if the pattern violates it, the arriving call is blocked.

With this formulation, the problem complexity grows exponentially with the number of channels; so we proposed an approach based on Hopfield neural networks, as described in the following section.

\section{HOPFIELD NEURAL NETWORKS}

The combinatorial optimization problem, i.e., finding a solution that minimizes a cost function and whose variables assume only two possible values, falls in the more general class of NP-complete (nondeterministic polynomial time complete) problems. Several complex methods have been proposed to find solutions to NP-problems [4].

Of particular interest is the neural network approach formulated by Hopfield and Tank [5]-[7], claimed to be a method that could be realized by hardware circuits with response times much shorter than those of other algorithmic methods.

Nevertheless, the original formulation suffered from a tendency to produce nonfeasible solutions called "spurious states."

Abe [8] proposed a modification to the original HopfieldTank's approach [5]-[7] to eliminate all the spurious states from the solution space. This type of neural network has been used in performing our simulations.

Let the energy function be defined as

$$
E=\frac{1}{2} x^{\prime} T x+b^{\prime} x
$$

where $T$ is a symmetric $n \times n$ matrix, $\bar{x}$ is an $n$-elements vector representing our variable, and $b$ is a constant $n$-elements vector of inputs.

The problem is equivalent to finding $x$ in order to minimize $E$ under the constraint that every single element of $x$ can take the final value 0 or 1 .

The value of each $x_{i}$-the $i$ th component of $\bar{x}$-falls into the real interval $[0,1]$.

Considering $x_{i}$ as the output of the $i$ th neuron of the network, the internal state $u_{i}$ is introduced, for which

$$
x_{i}=f\left(u_{i}\right) ; \quad i=1 \cdots n
$$

where $f\left(u_{i}\right)$ is a monotone nonlinear function; for instance, Hopfield suggests a hyperbolic tangent function $f\left(u_{i}\right)=$ $\frac{1}{2}\left[1+\tanh \left(u_{i}\right)\right]$. If we consider the energy function

$$
\tilde{E}=E-\sum_{i} \int_{0.5}^{x_{i}} f^{-1}(X) d X
$$

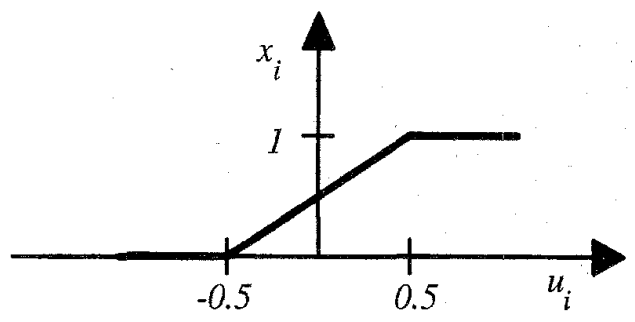

Fig. 4. Output transfer function of the neuron.

the time derivative of such a function is

$$
\begin{aligned}
\frac{d \tilde{E}}{d t} & =\sum_{i, j} T_{i, j} x_{j} \frac{d x_{i}}{d t}+\sum_{i} b_{i} \frac{d x_{i}}{d t}-\sum_{i} u_{i} \frac{d x_{i}}{d t} \\
& =\sum_{i} \frac{d x_{i}}{d t}\left(\sum_{j} T_{i, j} x_{j}+b_{i}-u_{i}\right)
\end{aligned}
$$

thus by considering a system for which

$$
\frac{d u_{i}}{d t}=-\sum_{j} T_{i, j} x_{j}-b_{i}-u_{i}
$$

we have

$$
\begin{aligned}
\frac{d \tilde{E}}{d t} & =-\sum_{i} \frac{d x_{i}}{d t} \frac{d u_{i}}{d t} \\
& =-\sum_{i}\left(\frac{d u_{i}}{d t}\right)^{2} \frac{d x_{i}}{d u_{i}} \leq 0
\end{aligned}
$$

for the monotonicity of $x=f(u)$. So $\tilde{E}$ is a Lyapunov function for (4)

$$
\frac{d \tilde{E}}{d t} \leq 0
$$

confirming that the set of (4) leads $E$ toward lower values; so for an arbitrary initial internal state $u_{0}$, a local minimum of the energy is achieved.

Under the hypothesis of using a piecewise-linear function $f(u)$, as shown in Fig. 4, the system (4) becomes a linear system operating on a closed hypercube

$$
\frac{d x}{d t}=-T x-b \quad 0 \leq x_{i} \leq 1 \quad i=1 \cdots n
$$

It is proved that no equilibrium points are found on the surfaces of the hypercube except for its vertexes. So it is possible to make all the feasible solutions be the only stable points [8].

The example in Fig. 5 shows the time evolution of a three-neuron Hopfield network whose stability points are the vertexes marked by a point in the state-space $\left(X_{1}, X_{2}, X_{3}\right)$.

It is not difficult to show that $E$ in (1) can be expressed as a quadratic form of the variables $V_{i^{*}, j}$ and if we call $\bar{x}$ the vector whose components are $V_{i^{*}, j}$ with $j=1 \cdots C H$ for a 


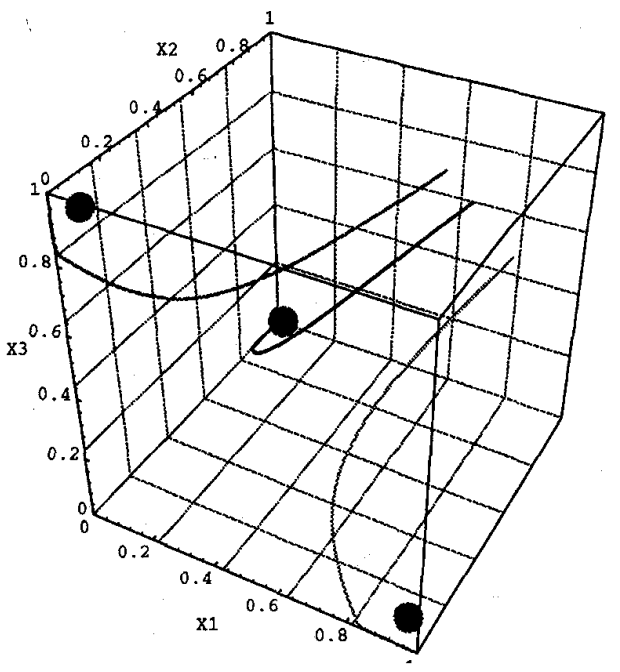

Fig. 5. Three-neurons time evolution in the state space.

fixed $i^{*}$, expression (2) represents (1) with

$$
\begin{aligned}
T_{j, j^{\prime}}= & B \\
b_{j}= & \frac{A}{2} \cdot \sum_{i} A_{i, j} \cdot \operatorname{Interf}\left(i, i^{*}\right) \\
& -B \cdot \operatorname{Traf}\left(i^{*}\right) \\
& -\frac{C}{2} \cdot \sum_{i} A_{i, j} \frac{\left(1-\delta_{i, i^{*}}\right)}{\operatorname{Dist}\left(i, i^{*}\right)}\left[1-\operatorname{Interf}\left(i, i^{*}\right)\right] \\
& -\frac{D}{2} \cdot A_{i^{*}, j} \\
& +\frac{F}{2} \cdot \sum_{i} A_{i, j}\left(1-\delta_{i, i^{*}}\right)\left[1-\operatorname{Res}\left(i, i^{*}\right)\right]
\end{aligned}
$$

where $\delta_{i j}$ is the Kronecker delta.

In order to make the system (6) stable for every possible $T$, we make the following substitutions without changing the values of $E$ at the vertexes of the hypercubic domain.

$$
\begin{aligned}
T_{j, j^{\prime}} & \rightarrow 0 \quad \forall j \\
b_{j} & \rightarrow b_{j}+\frac{T_{j, j^{\prime}}^{\text {(old })}}{2} .
\end{aligned}
$$

On the border of the hypercube we can find vertexes and nonvertexes points. The former are characterized by the fact that their components have only a value of 0 or 1 . The latter have at least one component in the interval $[0,1]$. Among the vertexes, we can see both the feasible solutions, which are compatible with the reuse and the channel demand constraints, and the nonfeasible ones.

The next step is to make all the feasible solutions be stable points of system (6).

Let $c$ be a vertex and $c(j)$ an adjacent vertex obtained by substituting the $j$ th component of $\mathbf{c}$ with its complement to one.

If $\mathrm{c}$ represents a feasible solution and $E(\mathrm{c})$ is its energy value, in order to make c a strongly stable point, we have to find values for constants $A, B, C, D$, and $F$ for which $E[\mathbf{c}(j)]>E(\mathbf{c})$ for every $j$.
TABLE I

DECISION RULES

\begin{tabular}{c|c|l}
\hline Rule: & Constant: & Description: \\
\hline R1 & A & no interference \\
\hline R2 & B & channel demand satisfied \\
\hline R3 & C & reuse packing preferred \\
\hline R4 & D & minimum variation from previous assignment preferred \\
\hline R5 & F & resonance condition preferred \\
\hline
\end{tabular}

We decide whether a vertex represents a feasible solution on the basis of five rules, as shown in Table I.

We start from a generic solution $c$; then, we change one (or more) of its components from 0 to 1 or vice versa. Due to the priorities of our rules, we are interested in the four following types of transitions:

- Transitions that violate rule R1, but lower energy value for rules R2, R3, R4, and R5,

- Transitions that increase energy for R2, but lower energy for R3, R4, and R5 leaving the term associated with R1 unchanged,

- Transitions that increase energy for R5, but lower energy for R3, R4, leaving the terms associated with R1 and R2 unchanged,

- Transitions that increase energy for R3, but lower energy for R4 leaving the terms associated with R1, R2, and R5 unchanged.

Since system (6) tends to lower the energy during its evolution we have to find some relations among the constant terms of (1) so that each of the above named transitions coincides with an energy increment. The following relations make all our feasible solutions be the only stable points in the system:

$$
\left\{\begin{array}{l}
C>D\left(\frac{1}{d_{1}}-\frac{1}{d_{2}}\right)^{-1} \\
E>\frac{C}{d_{\text {reuse }}}\left(\left[\frac{n_{\text {cells }}}{k_{\text {reuse }}}\right]-1\right)+D \\
B>E\left[\frac{n_{\text {cells }}}{k_{\text {reuse }}}\right] \\
B>\frac{C}{d_{\text {reuse }}}\left(\left[\frac{n_{\text {cells }}}{k_{\text {reuse }}}\right]-1\right)+D \\
A>B\left(2 n_{\text {channels }}+1\right)+\frac{C}{d_{\text {reuse }}}\left(\left[\frac{n_{\text {cells }}}{k_{\text {reuse }}}\right]-1\right)+D .
\end{array}\right.
$$

In (11) $d_{\text {reuse }}$ is the reuse distance, and $k_{\text {reuse }}$ denotes the reuse factor related to the reuse pattern of the cellular network.

In order to simulate the behavior of our neural network, we built a software model of the network using Euler's method to solve the set of differential of (6).

Let $x_{i}$ be the $i$ th component of the state vector $\bar{x}$, and $x_{i}^{\prime}$ the $i$ th component of the state derivative vector $\bar{x}^{\prime}$. After one time step $\Delta t$ we have

$$
\begin{aligned}
x_{i}^{\prime} & =x_{i}-\Delta t\left\{T_{i} \cdot \bar{x}+b_{i}\right\} \\
\text { New } \quad x_{i} & =\left\{\begin{array}{cl}
x_{i}^{\prime} & \text { for } 0<x_{i}^{\prime}<1 \\
1 & \text { for } x_{i}^{\prime} \geq 1 \\
0 & \text { for } x_{i}^{\prime} \leq 0
\end{array}\right.
\end{aligned}
$$




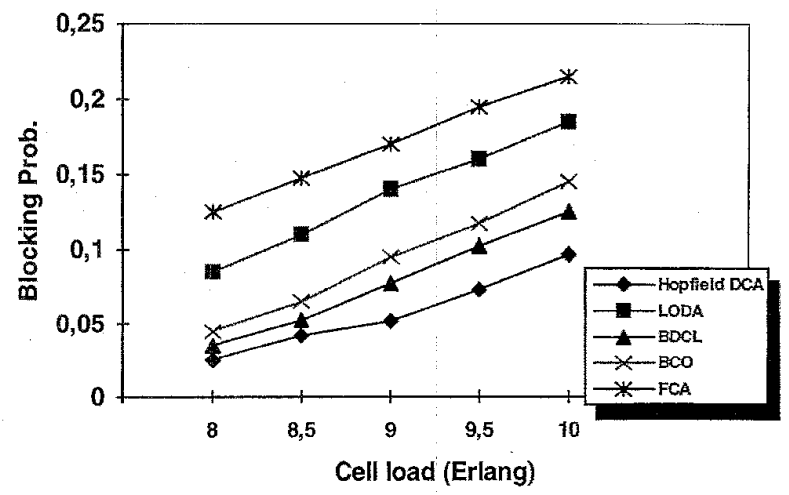

Fig. 6. Performance with uniform traffic.

where

$$
\begin{aligned}
\Delta t & =\frac{R}{\max _{i}\left\{\left(\frac{1}{2} \dot{T} \cdot \overline{1}+b\right)_{i}\right\}} \\
\overline{1} & =[1,1,1, \cdots, 1]^{\prime} .
\end{aligned}
$$

For the initial value of $x$, we chose $x_{i}(0)=0.5+r_{i}$, where $r_{i}$ is a uniformly distributed pseudo-random value in the range $[-\alpha / 2, \alpha / 2]$.

In our simulation, we chose a value of $R=0.3$ and $\alpha=10^{-9}$.

\section{Simulations AND PERFormanCE EVAluation}

The cellular network used by our simulations is made by a $7 \times 7$ portion of a hexagonal cell layout. We considered two rings of interfering cells and 70 channels available to the whole system. The performance of the channel allocation proposed in this paper has been derived in terms of the blocking probability for the incoming calls.

In the analytical model of a telephone system, the call arrivals are supposed to follow the Poisson process with the mean arrival rate of $\lambda$ (calls/min). The call duration is an exponentially distributed statistical process with mean $\bar{x}$ (min.). The quantity $\bar{x} \cdot \lambda=\rho$ expresses the load offered to the cellular network

We first computed the probability of refusing an incoming call for the nine central cells only and for a uniform traffic distribution among all the cells.

Under these simulation hypotheses, we were able to compare our results with those obtained by Zhang and Yum [10], [11] with the locally optimized dynamic assignment (LODA), borrowing with channel ordering (BCO), and borrowing with directional channel locking (BDCL) allocation algorithms.

Curves for theoretical FCA (ERLANG-B) are added to the numerical results obtained from the simulations.

In Fig. 6, the behavior of our technique is shown with respect to other DCA techniques for a uniform traffic distribution.

For a nonuniform traffic distribution, we considered the traffic patterns proposed by Zhang and Yum [10], [11], shown in Figs. 7 and 9.

The traffic load for each cell increased by a percent factor ranging from $0-140$. The simulation results are summarized in Fig. 8 for pattern $A$ and in Fig. 10 for pattern B.

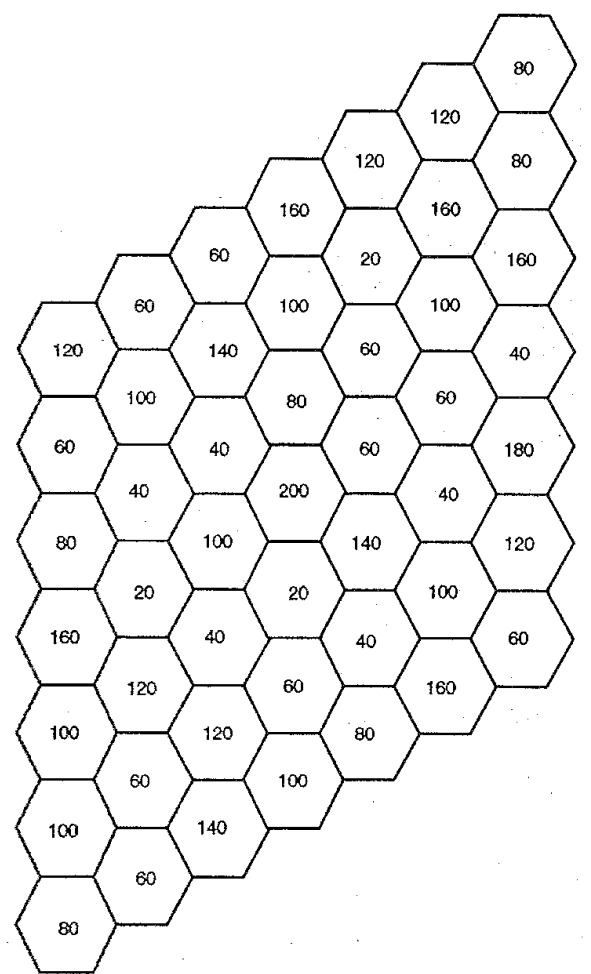

Fig. 7. Nonuniform traffic distribution (calls/hour)-pattern A.

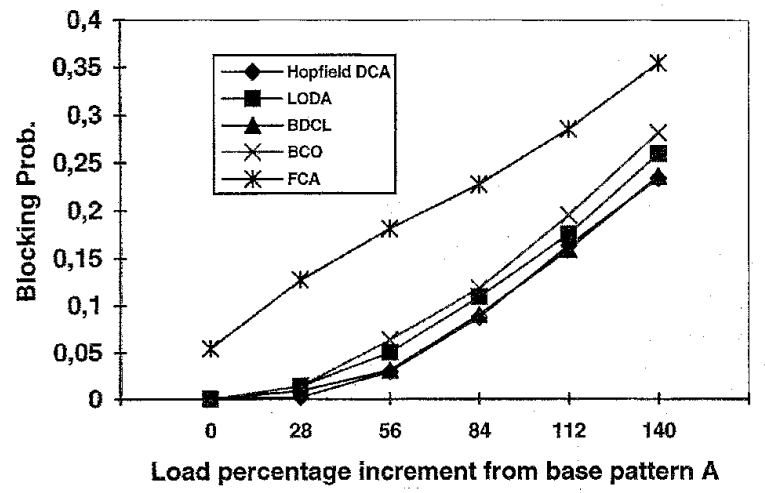

Fig. 8. Performance with nonuniform traffic load for pattern A.

Figs. 8 and 10 point out that the performance of the proposed Hopfield DCA technique is close to that of the BDCL technique [11]. In case of a uniform distribution, the neural allocator behaves even better than all the techniques considered in this comparison (see Fig. 6).

\section{CONCLUSION}

In quadratic optimization problems, Hopfield-Tank neural networks are not expected to perform much better than other methods for minima finding, since they effect a local search and the choice of a correct starting point is sometimes a hard task.

In 1991 a Hopfield-Tank neural approach to static channel assignment was developed by Kunz [12]. The needs of global 


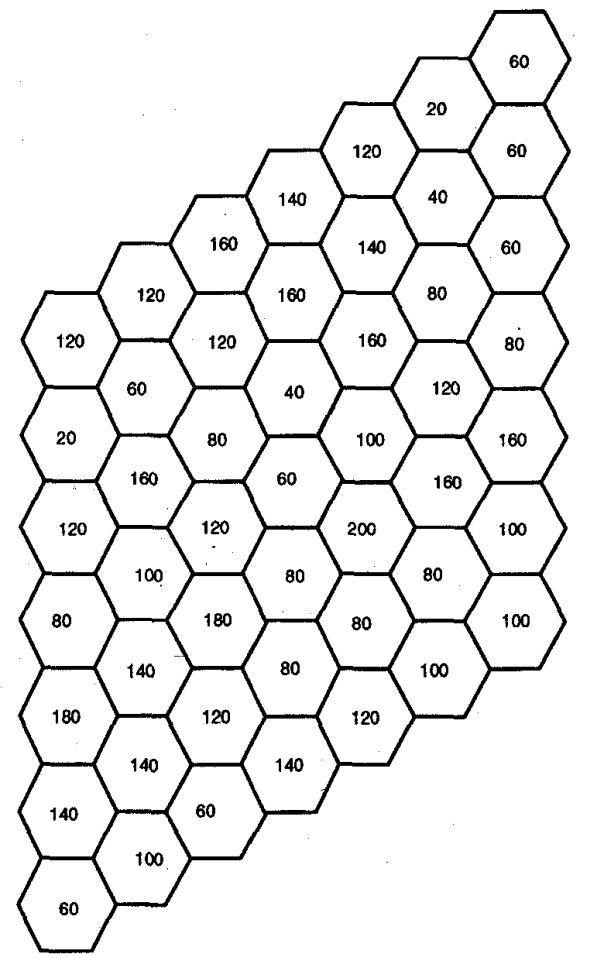

Fig. 9. Nonuniform traffic distribution (calls/hour)-pattern B.

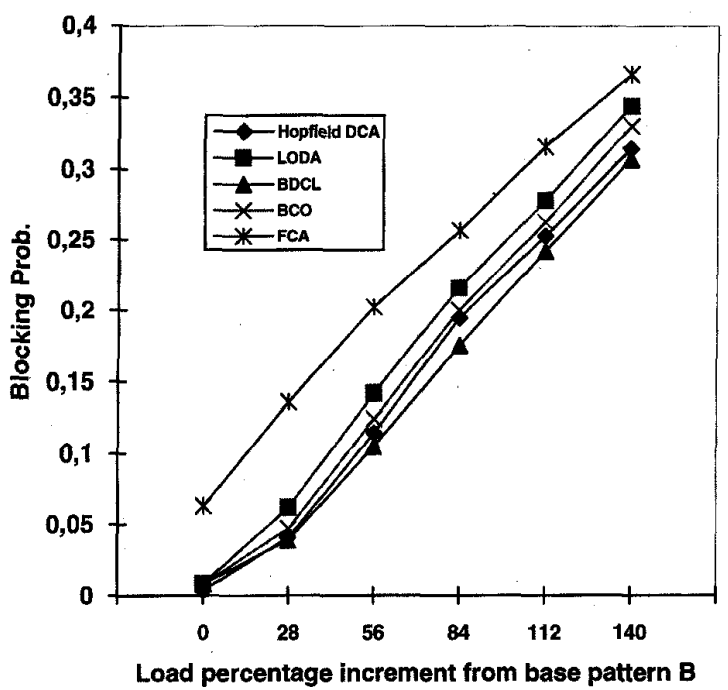

Fig. 10. Performance with nonuniform traffic load for pattern B.

minima solutions led Kunz to leave the Hopfield-Tank neural networks when he proved [13] that these networks in their original formulation were not able to find global minima of the energy function when applied to the allocation problem.

However, the problem of channel planning is quite different from that of dynamic channel assignment because of the absence of time constraints for finding the solution. Hence, the suboptimal solutions found by the Hopfield-Tank neural network, once deprived of the inconsistencies by Abe's method, seem to achieve a good level of performance, when compared with other DCA algorithms. This fact suggests that global finding is not so essential for the DCA problem if the average quality of the suboptimal solutions found is good. In a dynamic channel assignment, decision times are essential for the practical utilization of such techniques, and a neural approach like the one proposed in this paper seems to achieve a good level of performance with short processing times because of a massively parallel computational structure.

\section{REFERENCES}

[1] V. H. MacDonald, "Advanced mobile phone service: The cellular concept," Bell System Tech. J., vol. 58, no. 1, pp. 15-41, Jan 1979.

[2] W. C. Jakes, Jr., Microwave Mobile Communication. New York: Wiley, 1974

[3] P. A. Raymond, "Performance analysis of cellular networks," IEEE Trans. Comm., vol. 39, no. 12 , Dec. 1991

[4] S. Kirkpatrick, C. D. Gelatt, and M. P. De Vecchi, "Optimization by simulated annealing," Science, vol. 220, no. 4598, May 1983.

[5] J. J. Hopfield, "Neural networks and physical systems with emergent collective computational abilities," in Proc. Nat. Acad. Sci., 1982, vol. 79, pp. 2554-2558.

[6] those of two state neurons," in Proc. Nat. Acad. Sci., 1984, vol. 81, pp. 3088-3092.

[7] J. J. Hopfiled and D. W. Tank, "Neural' Computation of decisions in optimization problems," Biological Cybernetics, vol. 52, pp. 141-152, 1982.

[8] S. Abe, "Global convergence and suppression of spurious states of the Hopfield neural nets," IEEE Trans, Circuits Syst., vol. 40, no. 4, Apr. 1993

[9] J.-H. Li, A. N. Michel, and W. Porod, "Analysis and synthesis of a class of neural networks: Linear systems operating on a closed hypercube," IEEE Trans. Circuits Syst., vol. 36, no. 11, Nov. 1989.

[10] D. Everitt and D. Manfield, "Performance analysis of cellular mobile communication systems with dynamic channel assignment," JSAC, vol. 7 , no. 8 , Oct. 1989

[11] M. Zhang and T.-S. P. Yum, "Comparison of channel-assignment strategies in cellular mobile telephone systems," IEEE Trans. Veh. Technol., vol. 38, no. 4, Nov. 1989.

[12] D. Kunz, "Channel assignment for cellular radio using neural networks," IEEE Trans. Veh. Technol., vol. 40, no. 1, Nov. 1991.

[13] _ _ "Sub-optimal solutions obtained by the Hopfield-Tank neural network," Biolog. Cybernet., vol. 65, pp. 129-133, Nov. 1991.

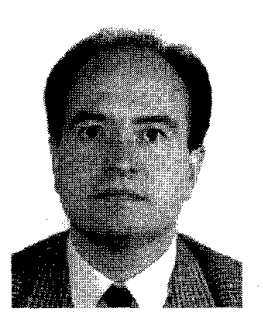

Enrico Del Re (M'78-SM'84) was born in Florence, Italy, in 1947. He received the Dr.Ing. degree in electronics engineering from the University of Pisa, Italy, in 1971.

Until 1975 he was engaged in public adminis tration and private firms, involved in the analysis and design of telecommunications, air traffic control equipment, and space systems. Since 1975 he has been with the Department of Electronics Engineering at the University of Florence, first as a Research Assistant, then as Associate Professor, and since 1986 as Professor. During the academic year 1987-1988, he was on leave from the University of Florence for a nine-month period of research at the European Space Research and Technology Center of the European Space Agency, Netherlands. His main research interest are digital signal processing, digital transmission techniques, and communication networks, on which he has published more than 100 papers in international journals and conferences. $\mathrm{He}$ is the coeditor of Satellite Integrated Communications Networks (NorthHolland, 1988) and an author of Data Compression and Error Control Techniques with Applications (Academic, 1985). He is Chairman of the European Project COST 227 "Integrated Space/Terrestrial Mobile Networks."

Dr. Del Re received the 1988-1989 premium from the IEE (U.K.) for the paper "Multicarrier Demodulator for Digital Satellite Communication Systems." $\mathrm{He}$ is a member of AEI, the European Association for Signal Processing (EURASIP), and the New York Academy of Sciences. He is included in Who's Who in the World. 


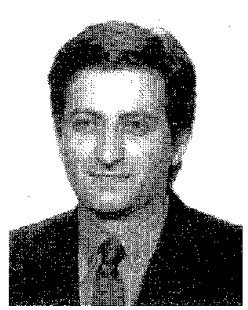

Romano Fantacci (S'84-M'88-SM'91) received the Dr.Ing. degree in electronics engineering from the University of Florence in Italy in 1982 and the $\mathrm{Ph} . \mathrm{D}$. in electronics engineering in 1987.

In 1982 he joined the Department of Electronics Engineering of the University of Florence first as a Research Assistant and presently as Associate Professor of Telecommunication Networks. His research interests include digital communications, queueing systems, multiple access systems, and personal communication networks.

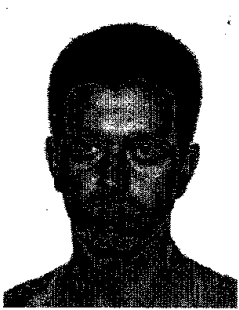

Luca Ronga (S'91-M'95) received the Dt.Ing. degree in electronics engineering from the University of Florence, Italy, in 1994.

$\mathrm{He}$ is a Research Assistant in Telecommunication Engineering at the University of Florence. His research interests cover neural elaborative models applied to telecommunications systems, digital transmission systems, and adaptive resources distribution for digital networks. 